\title{
Pedagogia y Didáctica
}

\section{UNA PROPUESTA PARA LA ENSEÑANZA DE LOS CONCEPTOS ÁCIDO-BASE, A PARTIR DEL ENFOQUE DIDÁCTICO: APRENDIZAJE POR INVESTIGACIÓN ${ }^{1}$}

\author{
Mónica Riveros Gutiérrez ${ }^{2}$ \\ dqu_mriveros133@pedagogica.edu.co \\ Pablo Gómez Meza ${ }^{2}$ \\ dqu_pgomez063@pedagogica.edu.co \\ Zendlli Villamil Triana ${ }^{2}$ \\ dqu_zvillamil573@pedagogica.edu.co
}

\section{Resumen}

El presente artículo corresponde a una propuesta para trabajar en la transformación de las ideas frente a los conceptos de ácido, base y neutralización, y su interpretación en las situaciones que involucren reacciones ácido-base, en un grupo de 30 estudiantes del curso 1101 del IED República de Colombia de la ciudad de Bogotá, Colombia. Los resultados obtenidos permiten inferir acerca de las relaciones entre ácido y $\mathrm{pH}$, así como la claridad de los conceptos base y ácido. Se propone una estrategia metodológica, basada en el modelo didáctico de aprendizaje por investigación, que consiste en el diseño de una estrategia de actividades, las cuales giran alrededor de una pregunta problema relacionada con el uso del producto conocido como "Alka-Seltzer" por su función de antiácido. Se busca con base en la estrategia aproximar a los estudiantes a los conceptos ácido-base en reacciones de neutralización.

Trabajo realizado en el marco del Seminario de Pedagogía y Didáctica III, durante el II-2012.

Estudiantes de Licenciatura en Química Universidad Pedagógica Nacional 


\section{Fundamentación Teórica}

Se entiende un problema como toda situación en la cual predomina la incertidumbre frente a la forma de actuar y/ o proceder (García \& García, 2000). La resolución de un problema implica la adquisición de una nueva información y por lo tanto según García y García, una evolución de las concepciones de la realidad que gira en torno a ese problema, por parte del sujeto que lleva a cabo tal acción; puede decirse que se aprende conforme se resuelven los problemas que se originan en un entorno diverso y cambiante.

Dado que no sólo es importante la resolución del problema, sino también los aspectos relativos a la búsqueda y reconocimiento del mismo, se plantea el modelo de Aprendizaje por Investigación, o el modelo de Investigación en el Aula, que según García y García (2000), permite partir de problemas notables de nuestro mundo real, y de ideas personales que los describen y los interpretan a través de un proceso reflexivo - crítico con otras ideas y con fenómenos naturales, generando un conocimiento escolar socializado y cooperado a través de procesos de cambio y evolución conceptual.

Si se plantea la investigación como un principio didáctico, es necesario que las estrategias de enseñanza tengan en cuenta la importancia de una actitud exploradora y curiosa dentro del proceso de enseñanza-aprendizaje, conforme a los principios constructivistas del aprendizaje, del aprendizaje significativo y de la importancia de la comunicación y la interacción social dentro de la construcción del conocimiento y del aprendizaje, y sin olvidar que estas estrategias no son de tipo "activo" puesto que los papeles del estudiante y el docente son igualmente importantes dentro del desarrollo de las mismas, y también, es necesario reconocer el peso que los contenidos temáticos tienen dentro de ellas.

Dentro del desarrollo de una estrategia para la resolución de problemas, una parte fundamental es la selección del problema a resolver; esto debe hacerse de manera consensuada entre el docente y los estudiantes, teniendo en cuenta, por un lado, los intereses de los estudiantes, y por otro, el nivel de enseñanza, la materia, la programación prevista, entre otros. (García \& García, 2000).
El aprendizaje cómo modelo didáctico debe poder responder a las preguntas ¿Qué enseñar? y ¿Cómo enseñar?, conforme a la interpretación que se tenga sobre la educación y la enseñanza, teniendo en cuenta que el aula de clase es un entorno con distintas configuraciones, donde hay interacción de diversas concepciones y formas de construcción de conocimiento, mediadas por variables comunicativas y contextuales. Por tanto, García y García (2000), expresan que es necesario un planteamiento que permita el tratamiento de los problemas inherentes a la propia dinámica comunicativa del aula como el de aquellos problemas que facilitan el aprendizaje del alumno; y el currículo debe ser entendido como un plan de actuación modificable, que proporciona al profesor unos fundamentos para proyectar, evaluar y justificar el proyecto educativo; el programa guía de actividades, aparece como la concreción en la práctica de la interacción entre los diferentes elementos.

\section{Metodología}

Para la formulación de la estrategia didáctica es necesario realizar una revisión bibliográfica acerca del modelo de Aprendizaje por Investigación, conforme a los planteamientos de García y García (2000), Gil y Martínez (1987).

La estrategia de actividades retoma los planteamientos de Gil y Martínez (1987), buscando favorecer mediante una serie de actividades, que los alumnos puedan construir y afianzar conocimientos al tiempo que se familiarizan con algunas características del trabajo científico. Por ello, exige que el conjunto de actividades posean una lógica interna que evite aprendizajes descontextualizados, y procesos excesivamente erráticos.

Furió y Gil (1978), citado en Gil y Martínez (1987), enmarcan que dentro del programa guía de actividades (PGA) se destacan tres grandes grupos de actividades que fundamentan el mismo, denominadas respectivamente de iniciación, de desarrollo y de acabado del tema. Para realizar el correspondiente PGA se partió de la siguiente pregunta de investigación: 


\section{¿Por qué el alka-seltzer se utiliza como medicamento para la acidez?}

Igualmente, para el planteamiento de las diferentes actividades se esbozan una serie de competencias que determinan el fundamento y las finalidades de las actividades propuestas, como se muestra en el siguiente cuadro:

\begin{tabular}{|c|c|}
\hline $\begin{array}{c}\text { COMPETENCIA BÁSICA QUE SE } \\
\text { PRETENDE PROMOVER }\end{array}$ & \multicolumn{1}{c|}{ DESCRIPCIÓN } \\
\hline Interpretar & $\begin{array}{l}\text { - Comprender reacciones ácido-base a nivel teórico } \\
\text { (lápiz y papel) como a nivel experimental (trabajo } \\
\text { de laboratorio). } \\
\text { - Identificar la aplicación de reacciones ácido-base } \\
\text { en aspectos cotidianos. }\end{array}$ \\
\hline Proponer & $\begin{array}{l}\text { Alproponer, el estudiante muestra que ha empezado } \\
\text { a comprender claramente los conceptos. }\end{array}$ \\
\hline Argumentar & $\begin{array}{l}\text { - Cuando el estudiante propone, y a la vez, tiene los } \\
\text { argumentos suficientes para defender su punto de } \\
\text { vista ha generado relaciones que puede aplicar a } \\
\text { diferentes contextos. }\end{array}$ \\
\hline
\end{tabular}

Para el planteamiento de las actividades se tienen en cuenta tres fases:

- Fase de Iniciación: A partir de ella, se busca aproximar a los estudiantes de una forma dinámica a los conceptos inicialmente propuestos, generando un interés de los mismos por la temática. De igual forma, a través de esta fase se pretende examinar las ideas previas que los estudiantes poseen acerca de los conceptos ácido-base.

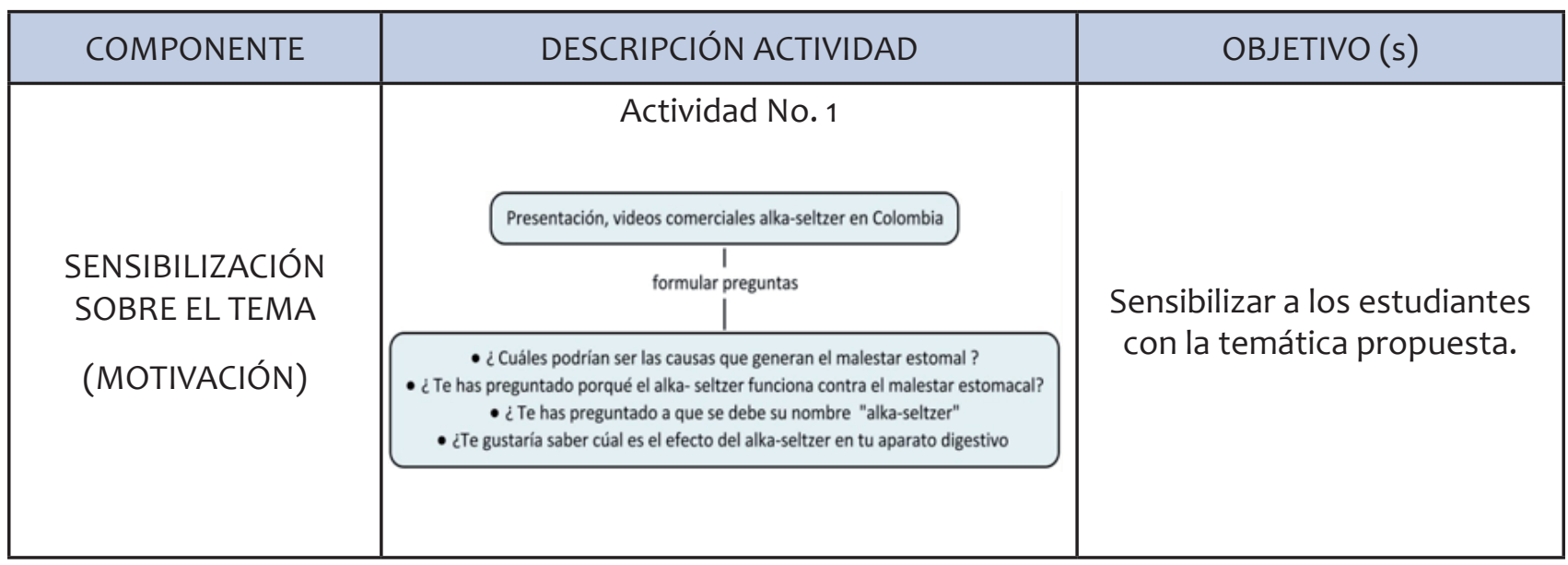




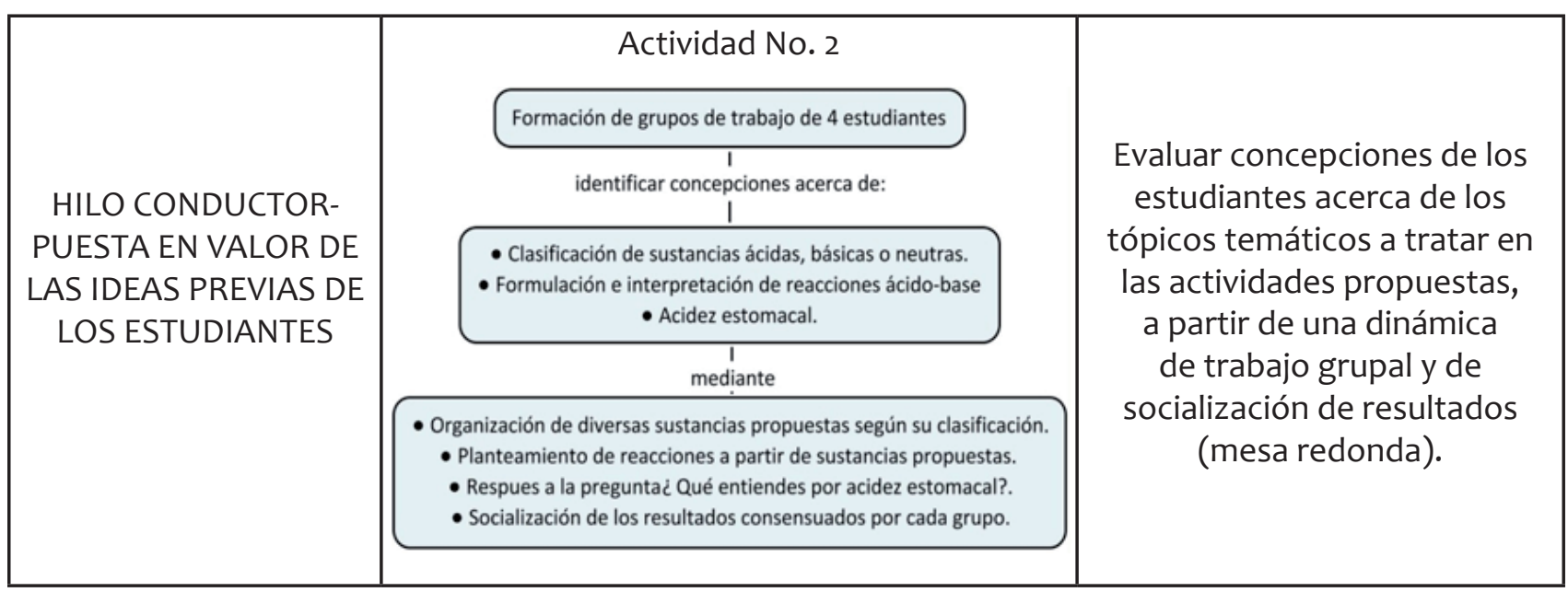

Cuadro No. 1. Exposición de las actividades relacionadas en la fase de iniciación del PGA

- Fase de Desarrollo: En la etapa de desarrollo se proponen una serie de actividades que permiten una construcción significativa y un dominio apropiado de los conceptos ácido y base, por parte de los estudiantes, y en particular, cuando dichos conceptos se aplican al modo de acción del alka-seltzer.

\begin{tabular}{|c|c|c|}
\hline COMPONENTE & DESCRIPCIÓN ACTIVIDAD & OBJETIVO \\
\hline $\begin{array}{l}\text { INTRODUCCIÓN DE } \\
\text { CONCEPTOS }\end{array}$ & \begin{tabular}{|c|} 
Actividad No. 3 \\
$\begin{array}{c}\text { Consulta de: Página web acerca de la acidez } \\
\text { estomacal; página web principal del alka-seltzer e información sobre } \\
\text { teorías ácido-base. }\end{array}$ \\
- Responder una serie de preguntas las cuales serán tenidas en cuenta \\
para la actividad posterior.
\end{tabular} & $\begin{array}{l}\text { Suministrar la información } \\
\text { necesaria para llevar a cabo una } \\
\text { conceptualización acerca de los } \\
\text { tópicos temáticos. } \\
\text { Propiciar la relación de conceptos } \\
\text { a partir de la información } \\
\text { suministrada. }\end{array}$ \\
\hline $\begin{array}{l}\text { CONSTRUCCIÓN Y } \\
\text { MANEJO SIGNIFICATIVO } \\
\text { DE LOS CONCEPTOS }\end{array}$ & $\begin{array}{l}\text { Actividad No. } 4 \\
\text { Se socializan las respuestas a las preguntas } \\
\text { que se formularon en la actividad anterior; } \\
\text { se busca un consenso entre las diversas } \\
\text { respuestas por mediación de las explicaciones } \\
\text { y aclaraciones que el profesor realice y crea } \\
\text { pertinentes. }\end{array}$ & $\begin{array}{l}\text { Generar un espacio que fomente } \\
\text { la construcción colectiva del } \\
\text { conocimiento, y su validación por } \\
\text { parte del profesor. }\end{array}$ \\
\hline
\end{tabular}




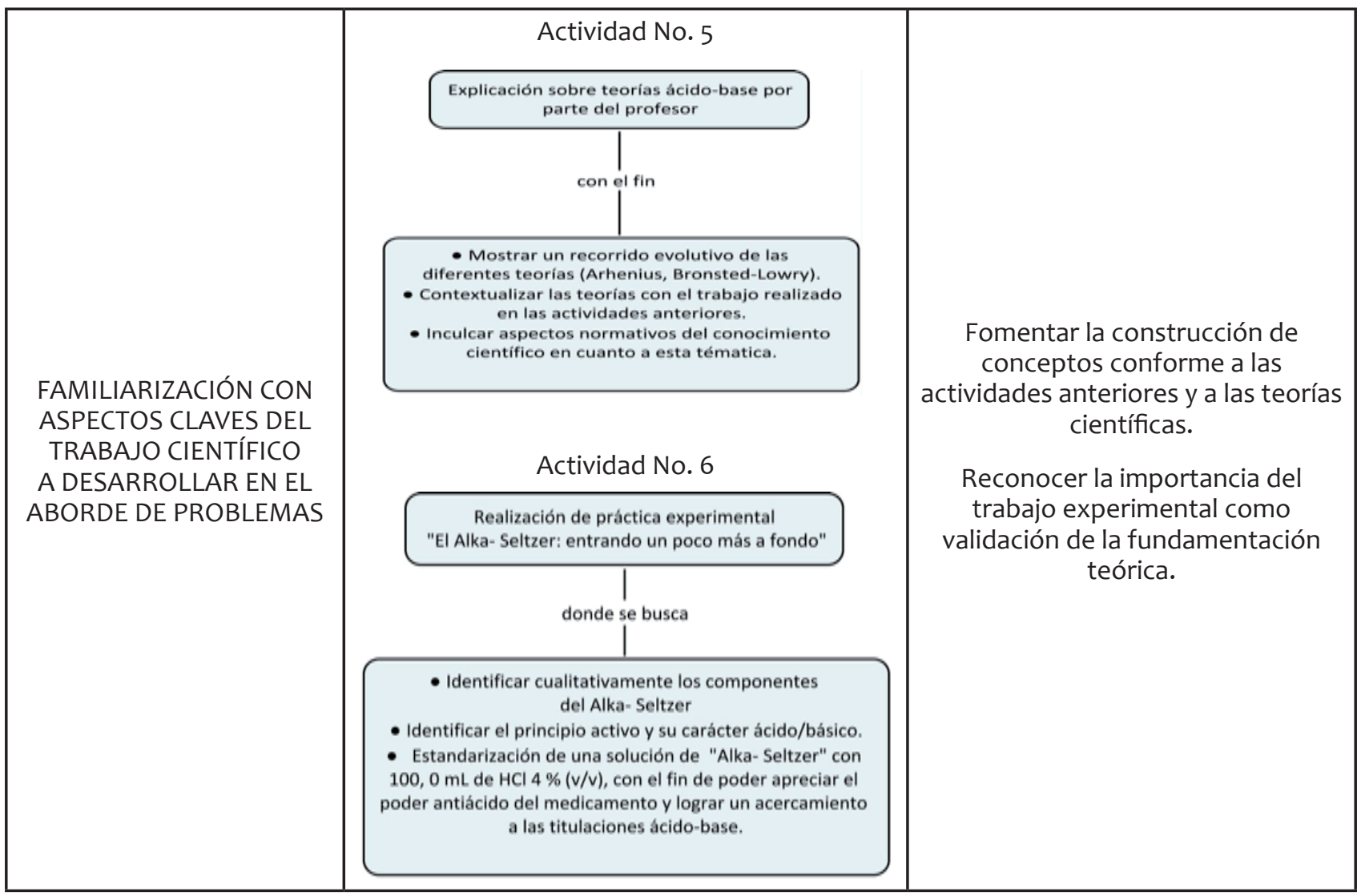

Cuadro No. 2. Exposición de las actividades relacionadas con la fase de desarrollo del PGA

- Fase de Acabado: En esta fase se plantean una serie de instrumentos que permiten evaluar la construcción de los conocimientos, capacidades y actitudes con respecto a la temática planteada. Cabe aclarar, que esta fase no se realiza como un proceso terminal, sino que es de gran relevancia llevarlo a cabo durante todo el proceso. Para esta parte se recomienda:

\begin{tabular}{|c|c|c|}
\hline COMPONENTE & DESCRIPCIÓN ACTIVIDAD & OBJETIVOS \\
\hline Evaluación de contenidos & $\begin{array}{c}\text { Taller de Finalización: Se proponen } \\
\text { diversos ejercicios y preguntas } \\
\text { problema cuyo fin es evaluar los } \\
\text { conceptos y/o conocimientos } \\
\text { aprendidos durante la secuencia. }\end{array}$ & $\begin{array}{c}\text { Aplicar los conocimientos aprendidos } \\
\text { a la comprensión del fundamento del } \\
\text { alka-selter, y a su vez, su aplicación en } \\
\text { diferentes contextos. }\end{array}$ \\
\hline Evaluación de la propuesta & $\begin{array}{c}\text { Percepciones de la propuesta: Se } \\
\text { someterá la estrategia planteada } \\
\text { a evaluación por los mismos } \\
\text { estudiantes, mediante una } \\
\text { socialización. }\end{array}$ & $\begin{array}{c}\text { Conocer las opiniones de lostudiantes } \\
\text { acerca de su experiencia durante } \\
\text { la realización de la propuesta, para } \\
\text { determinar qué aprendieron, qué no } \\
\text { aprendieron, qué aspectos consideran } \\
\text { que tiene a favor y qué se puede mejorar }\end{array}$ \\
\hline
\end{tabular}

Cuadro No. 3. Exposición de las actividades relacionadas con la fase de acabado del PGA. 
Implicaciones pedagógicas y didacticas de la implementación del modelo aprendizaje por investigación dirigida en la enseñanza de la Química.

En este mundo donde el conocimiento es fuente inicial del progreso, se convierte en meta esencial del ser humano ubicar a la naturaleza y al hombre mismo como objeto de estudio; es por esto que, según Porlán (1997), ha surgido el estudio del mundo físico en relación con el mundo de las ideas.

Así pues, el docente tiene un papel fundamental en la sociedad promoviendo el análisis de esta relación, buscando que el sujeto encuentre la motivación hacia conocer el mundo, su funcionamiento y sus relaciones. En este proceso debe estar implícito el conocimiento didáctico, como lo expresa Porlán, en su libro, "Constructivismo y Escuela. Hacia un modelo de enseñanza-aprendizaje basado en la investigación.". Desde este punto de vista, el profesor ya no hace parte de la construcción del conocimiento, sino que también puede ser investigador; así lo ve Elliott (1980, citado en Porlan, 1997). Desde estas miradas, Lowyck (1983 citado en Porlán, 1997), hace una reflexión acerca del carácter que presenta en el aula el docente, ya que no debería ser lineado por teorías limitadas en relaciones unidireccionales de docente y alumno, debido a que los procesos de enseñanza aprendizaje son intencionales, puesto que no todo el ambiente está proporcionado de la misma manera. Por ende, se pretende que el profesor sea reflexivo con su propio accionar, y no impulse en el alumno la resolución de cada problema desde una operación mecánica, sino que dirija este proceso adecuando la situación a un entorno más comprensible para éste.

En cuanto al alumno, es importante reconocerlo como parte del proceso de trasformación del actuar y constructor de sus esquemas mentales (Porlán, 1997) . El siguiente esquema explica, a grandes rasgos, el proceso que experimenta el alumno en cuanto al aprendizaje de las ciencias durante la enseñanza formal.
Esquema No. 1. Constructivismo en el pensamiento del alumno.

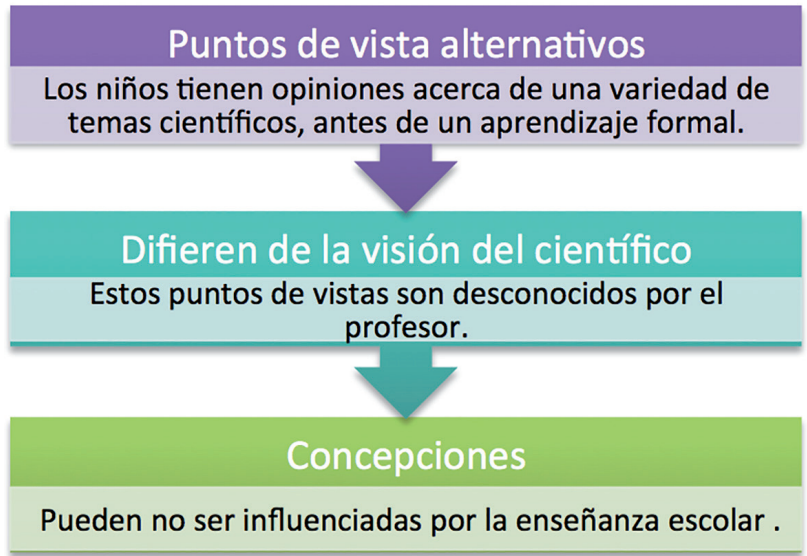

Sin embargo, como lo expresa Porlán, el solo centrarse en el constructo cognitivo de los docentes y alumnos, evade las situaciones del entorno en donde se presentan los procesos de enseñanza-aprendizaje, olvidando la motivación y el interés. Por ende, Doyle $(1981,1985,1986$, citado en Porlán, 1997) afirma que el accionar del docente y del estudiante están determinados por la reciprocidad que se establece en el aula, generando una estructura de poder, y a su vez influyendo en su vida psico-social. En este intercambio, el saber escolar es influenciado por el flujo de comunicación en el aula, construyéndose mediante la integración grupal, desde actividades y discusiones expuestas.

En el caso particular de la química es posible aprovechar este modelo articulándolo al campo didáctico de la Química Cotidiana como se puede encontrar en trabajos tales como los propuesto por Jiménez Liso y De Manuel (2009), los cuales realizan estudios didácticos de diversos trabajos que se presentan en ferias de ciencia y en programas de televisión. Estos autores plantean la necesidad de una proximidad a un contexto para el alumnado así como selección de contenidos necesarios que puedan ayudar al análisis o la transformación de las actividades divulgadas, ya sea a través de ferias, congresos y programas de televisión (y esto se puede llevar a otros escenarios educativos, como el aula de clase en sí) para superar el conocido rechazo de la química por su abstracción, dificultad, o desco- 
nexión con lo cercano al alumnado. (Jimenez-Liso y De Manuel, 2009). Partiendo de este supuesto, es necesario generar estrategias que permitan un acercamiento entre ese conocimiento químico que debe enseñarse en el aula y el diario vivir de un estudiante; podría considerarse que una manera para llegar a esto sería buscando la aproximación al conocimiento a través de formulaciones de los estudiantes, y a pesar de que lograr la formulación de un problema de investigación por parte del alumno es un hecho difícil, sería ahí donde el papel del profesor toma un valor importante, pues estaría encargado, por un lado, de problematizar algún aspecto de la cotidianidad o del entorno ambiental del estudiante (García y García, 2000) y con base en ello, diseñar una estrategia completamente innovadora para la enseñanza de contenidos químicos en un aula mediada por un contexto determinado.

El entorno educativo está mediado por multiplicidad de esferas contextuales, las cuales actúan de manera cercana, intima o distante pero nunca indiferente con el desarrollo sujeto que se educa (Bronfenbenner, 1979 citado por SED 2009). Partir de este supuesto, podría ayudar a generar estrategias investigativas bastante particulares, pero sobre todo, podría despertar el interés en los estudiantes por acercarse a aquello que usualmente no se acercan, generalmente mediado por la enseñanza, y que de una u otra manera puede ser de gran utilidad para su vida, a pesar de que ellos no lo crean.

\section{Consideraciones finales}

Se hace relevante desarrollar e implementar programas guías de actividades, ya que le permiten a los estudiantes comprender con mayor facilidad los conceptos y obtener un aprendizaje significativo con respecto a las diferentes temáticas trabajadas. Asimismo, es importante que los programas propuestos se estructuren a partir de investigaciones previas, para que este tipo de propuestas presenten una finalidad y sean significativas a nivel educativo.

Por otro lado, se considera relevante trabajar con el modelo por investigación dirigida, pues así estudiantes y profesores podrán trabajar desde situaciones de su entorno más próximo, generando un conocimiento significativo y contextualizado para el alumno.

\section{Bibliografía}

García, J., \& García, F. (2000). Aprender investigando: Una propuesta metodológica basada en la investigación. Sevilla: Díada editorial.

Gil, D., \& Martinéz, J. (1987). Los programas-guía de actividades: una concreción del modelo constructivista de aprendizaje de las ciencias. Investigación en la Escuela (3). 3-12.

Jimenez-Lizo, M. \& De Manuel, E. (2009). La Química cotidiana. Una oportunidad para el desarrollo profesional del profesorado. Revista Electrónica de Enseñanza de las Ciencias. VII (3), 878-900.
Porlán, R. (1997). Constructivismo y Escuela. Hacia un modelo de enseñanza-aprendizaje basado en la investigación. (Cuarta edición ed.). Sevilla: Díada editora.

Secretaría de Educación Distrital-SED (2009). Caracterización. Documento Segunda Jornada de Formación. Reorganización de la Enseñanza por ciclos y periodos académicos. 